\title{
Correction to: Fine-tuning Flowering Time via Genome Editing of Upstream Open Reading Frames of Heading Date 2 in Rice
}

Xinxin Liu ${ }^{1,2+}$, Hualong Liu ${ }^{3+}$, Yuanye Zhang ${ }^{4 \dagger}$, Mingliang He ${ }^{2,5}$, Rongtian $\mathrm{Li}^{4}$, Wei Meng ${ }^{1}$, Zhenyu Wang ${ }^{2}$, Xiufeng $\mathrm{Li}^{\mathrm{i}^{*}}$ and Qingyun $\mathrm{Bu}^{2^{*}}$

\section{Correction to: Rice 14, 59 (2021)}

https://doi.org/10.1186/s12284-021-00504-w

It was highlighted that in the original article (Liu et al. 2021) the affiliations were incorrect and presented as below. The original article has been updated.

\section{Incorrect Affiliations}

Xinxin Liu1†, Hualong Liu2†, Yuanye Zhang3+, Mingliang He4,5, Rongtian Li3, Wei Meng1, Zhenyu Wang4, Xiufeng $\mathrm{Li}^{4}$ " and Qingyun Bu4*

1College of Life Science, Northeast Forestry University, 150081 Harbin, China.

2College of Life Science, Heilongjiang University, 150080 Harbin, China.

3Northeast Institute of Geography and Agroecology, Key Laboratory of Soybean Molecular Design Breeding, Chinese Academy of Sciences, 150081 Harbin, China.

4Rice Research Institute, College of Agriculture, Northeast Agricultural University, 150030 Harbin, China.

5University of Chinese Academy of Sciences, Beijing 100049, China.

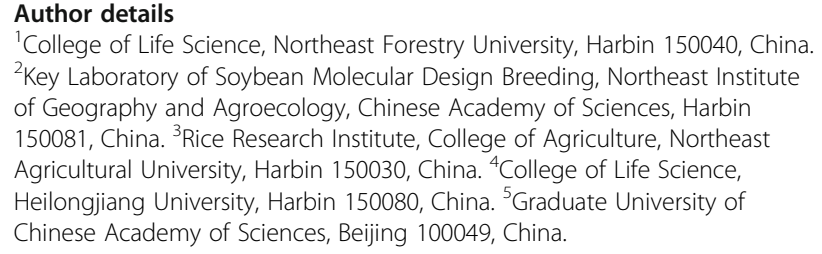

${ }^{1}$ College of Life Science, Northeast Forestry University, Harbin 150040, China. ${ }^{2}$ Key Laboratory of Soybean Molecular Design Breeding, Northeast Institute of Geography and Agroecology, Chinese Academy of Sciences, Harbin 150081, China. ${ }^{3}$ Rice Research Institute, College of Agriculture, Northeast Agricultural University, Harbin 150030, China. ${ }^{4}$ College of Life Science, Heilongjiang University, Harbin 150080, China. ${ }^{5}$ Graduate University of Chinese Academy of Sciences, Beijing 100049, China.

The original article can be found online at https://doi.org/10.1186/s12284021-00504-w.

* Correspondence: lixiufeng@iga.ac.cn; buqingyun@iga.ac.cn

${ }^{+}$Xinxin Liu, Hualong Liu and Yuanye Zhang contributed equally to this work.

${ }^{2}$ Key Laboratory of Soybean Molecular Design Breeding, Northeast Institute

of Geography and Agroecology, Chinese Academy of Sciences, Harbin

150081, China

Full list of author information is available at the end of the article
Published online: 20 July 2021

\section{Reference}

Liu et al (2021) Fine-tuning Flowering Time via Genome Editing of Upstream Open Reading Frames of Heading Date 2 in Rice. Rice 14:59. https://doi.org/1 0.1186/s12284-021-00504-w

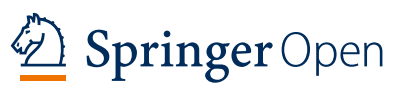

(-) The Author(s). 2021 Open Access This article is licensed under a Creative Commons Attribution 4.0 International License, which permits use, sharing, adaptation, distribution and reproduction in any medium or format, as long as you give appropriate credit to the original author(s) and the source, provide a link to the Creative Commons licence, and indicate if changes were made. The images or other third party material in this article are included in the article's Creative Commons licence, unless indicated otherwise in a credit line to the material. If material is not included in the article's Creative Commons licence and your intended use is not permitted by statutory regulation or exceeds the permitted use, you will need to obtain permission directly from the copyright holder. To view a copy of this licence, visit http://creativecommons.org/licenses/by/4.0/. 